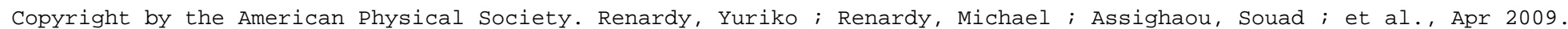
"Numerical simulation of drop retraction after a strain jump," PHYSICAL REVIEW E 79(4): 046323. DOI: 10.1103/

\title{
Numerical simulation of drop retraction after a strain jump
}

\author{
Yuriko Renardy and Michael Renardy* \\ Department of Mathematics, Virginia Tech, 460 McBryde Hall, Blacksburg, Virginia 24061-0123, USA \\ Souad Assighaou and Lazhar Benyahia ${ }^{\dagger}$ \\ Polyméres, Collö̈des, Interfaces, UMR CNRS 6120, Université du Maine 1, Avenue Olivier Messiaen, 72085 Le Mans, France
}

(Received 16 February 2009; published 24 April 2009)

\begin{abstract}
A spherical drop of one liquid suspended in another is subjected to an instantaneous initial shear. We simulate the retraction of the drop to its spherical equilibrium and compare the results with recent experiments. In agreement with the experiments, we observe a two-step relaxation process.
\end{abstract}

DOI: 10.1103/PhysRevE.79.046323

PACS number(s): 47.55.D-

\section{INTRODUCTION}

In recent experiments, Assighaou and Benyahia [1] investigated the retraction of a deformed liquid droplet suspended in another liquid. They subjected the drop to an initial step strain and then observed the retraction. If the initial deformation is large enough, the drop rapidly transitions from the initial ellipsoidal shape to a new shape which looks more like a cylinder with spherical caps. The subsequent relaxation is characterized by two phases. In the first phase, the drop maintains the shape of a cylinder with spherical caps; in the second phase it becomes ellipsoidal.

Each of the two phases has a characteristic relaxation time. The results in [1] are described in terms of the Hencky strain and a time scale based on capillary time. Let $\eta_{d}$ and $\eta_{m}$ denote the viscosities of the drop and matrix, $K=\eta_{d} / \eta_{m}$, $r_{0}$ the radius of the drop when it is a sphere, and $\Gamma$ the surface tension coefficient. If $L$ is the length of the deformed drop, the Hencky strain is defined by

$$
\gamma=\ln \frac{L}{2 r_{0}},
$$

and a capillary time scale is given by

$$
\tau_{C a}=\frac{\eta_{m} r_{0}}{\Gamma} .
$$

In the second phase of the relaxation, the behavior is determined by linear stability, and it is found that

$$
\gamma \sim \exp \left(-t / \tau_{2}\right)
$$

where

$$
\tau_{2}=\frac{\tau_{C a}}{4} \frac{(19 K+16)(2 K+3)}{10(K+1)} .
$$

In the first phase of the relaxation, on the other hand, the Hencky strain decreases linearly:

\footnotetext{
*renardy@math.vt.edu; URL: http://www.math.vt.edu/people/ renardyy

${ }^{\dagger}$ Lazhar.Benyahia@univ-lemans.fr; URL: http://www.univlemans.fr/sciences/wpci
}

$$
\gamma \sim-t / \tau_{1}
$$

where

$$
\tau_{1}=4.4 \tau_{2}
$$

This ratio of time scales is found to be independent of the initial strain and viscosity ratio. The transition between the two regimes occurs at a Hencky strain of order 0.34 ; this value is also independent of the initial strain and viscosity ratio.

Assighaou and Benyahia [1] attempted to offer a qualitative explanation for the observed behavior. A crucial hypothesis in this explanation is that the difference between the maximum and minimum of curvature on the surface of the drop decays exponentially in both phases of relaxation. Since the curvatures are not measured, they are not able to test this hypothesis directly.

In this paper, we present direct numerical simulations of the problem. We do not attempt to simulate the stretching of the drop at the beginning of the experiment and simply start the simulation with an initial drop shape arising from instantaneous affine deformation of a sphere, together with zero initial velocities. Theoretically, this assumption is justified for viscosity ratio 1 , if the initial deformation is sufficiently rapid, and other effects such as inertia or non-Newtonian effects are neglibible. For viscosity ratios other than 1, the initial deformation of the drop cannot be expected to be affine, since there is a discontinuity in shear rate at the interface.

For the simulation, we use the in-house VOF-PROST code (volume-of-fluid, paraboloid representation of the interface in the surface tension force), which is detailed in [2]. Briefly,

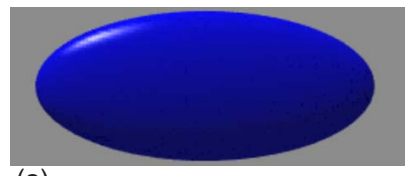

(a)

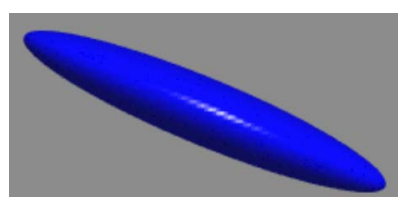

(b)
FIG. 1. (Color online) The initial shape for numerical simulations is an elongated ellipsoid. Here, $S=2, K=1, \tau_{C a}=0.56$, $\tau_{1} / \tau_{C a}=9.62$, and $\tau_{2} / \tau_{C a}=2.19$. (a) Top view shows an ellipse of width $2 r_{0}$ in the $y$ direction. (b) Side view. 


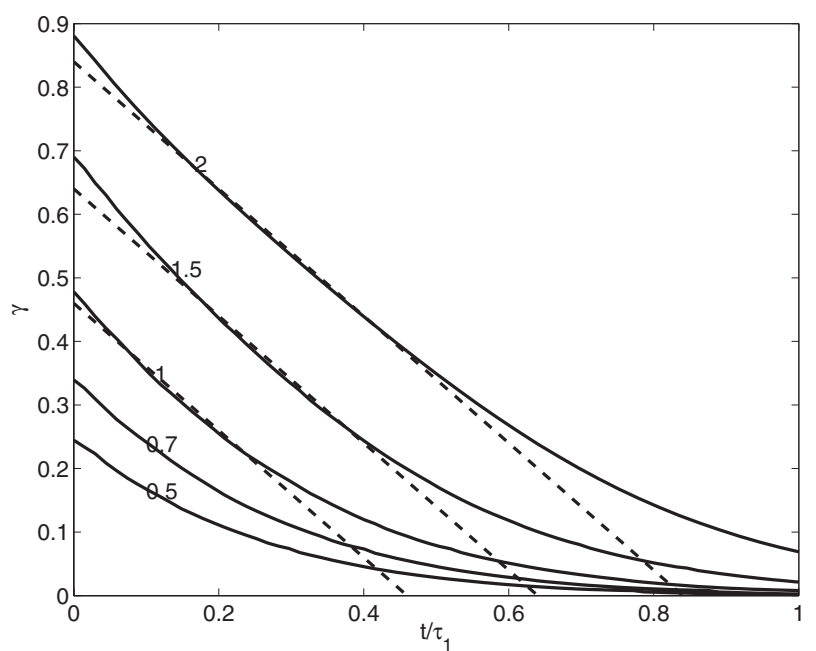

FIG. 2. Simulation results for $\gamma=\ln \left(L / 2 r_{0}\right)$ vs $t / \tau_{1}, K=1, \tau_{C a}$ $=0.35, \tau_{1} / \tau_{C a}=9.62$, and $\tau_{2} / \tau_{C a}=2.19$. Shear deformation values are noted on the plot; $S=0.5,0.7,1,1.5,2$. Lines with slope -1 are given for reference (- - -).

this is a finite difference code on a three-dimensional Cartesian mesh with a staggered grid. A volume-of-fluid method is used to track the interface [3] by using a color function to track the placement of the drop liquid and the matrix liquid. The full Navier-Stokes equations are solved with the continuum surface force algorithm for modeling the interfacial tension force [4]. The interface is reconstructed locally from a least-squares fit of paraboloids to the values of the color function in the interface cell and its neighbors, and this allows an accurate computation of curvatures. Figure 1 shows a sample initial drop shape.

In the simulations which follow, we have kept the viscosity ratio of the fluids equal to 1 , and we have chosen the densities sufficiently small that inertial effects are insignificant. A typical value of the Ohnesorge number in our computations, defined as $\eta / \sqrt{\rho \Gamma r_{0}}$, where $\rho$ denotes the density,

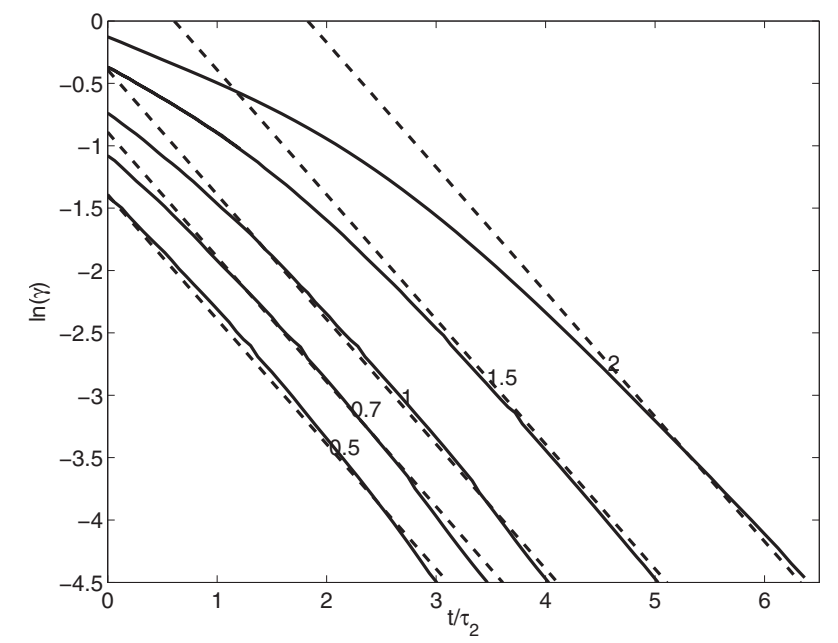

FIG. 3. Simulation results for $\ln (\gamma)=\ln \left[\ln \left(L / 2 r_{0}\right)\right]$ vs $t / \tau_{2}$. $K=1, \quad \tau_{C a}=0.35, \quad \tau_{1} / \tau_{C a}=9.62, \quad$ and $\quad \tau_{2} / \tau_{C a}=2.19$. $S=0.5,0.7,1,1.5,2$. Lines with slope -1 are given for reference $(--)$.

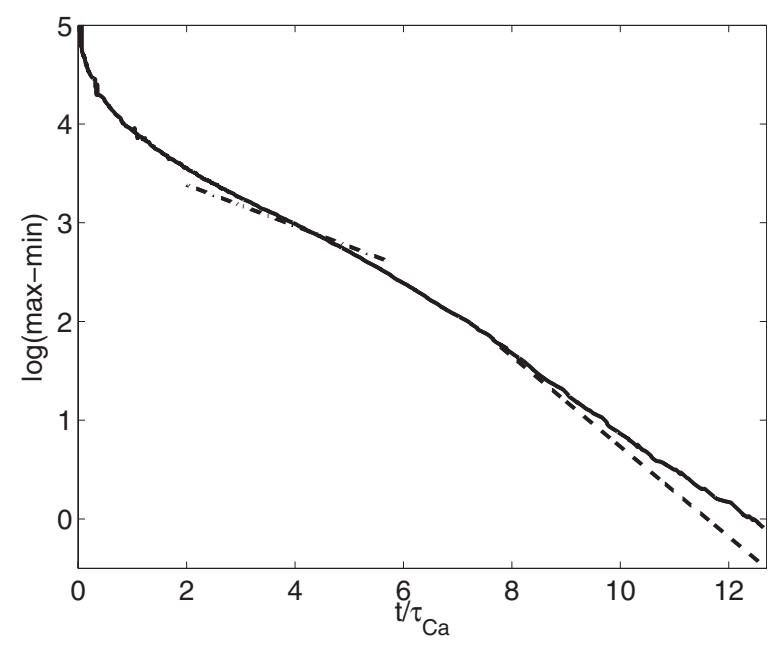

FIG. 4. Simulation results for $\ln (\max -\min )$ vs $t / \tau_{C a}$, where $\max$ and min refer to the curvature maximum and minimum. $K=1, \tau_{C a}$ $=0.35, \tau_{1} / \tau_{C a}=9.62, \tau_{2} / \tau_{C a}=2.19$, and $S=2$. Lines with slopes $-2 \tau_{C a} / \tau_{1}(-),. \tau_{C a} / \tau_{2}(-)$.

is about 3. The parameter $S$ denotes the initial shear of the drop, i.e., the initial shape is obtained from a sphere via the deformation $(x, y, z) \mapsto(x+S z, y, z)$.

\section{DIRECT NUMERICAL SIMULATION RESULTS}

Figures 2 and 3 show the evolution of the drop length. In the first figure, $\gamma$ is plotted against a time normalized with $\tau_{1}$. The dashed lines have slope -1 . We see that the lines fit the first stage of drop evolution reasonably well, in agreement with the experiments. We note that at the very beginning of the retraction process, the retraction is faster than the linear fit; we shall return to this point below.

Figure 3 shows $\ln (\gamma)$ plotted against a time normalized with $\tau_{2}$. We see that lines with slope -1 fit the later evolution

(a)
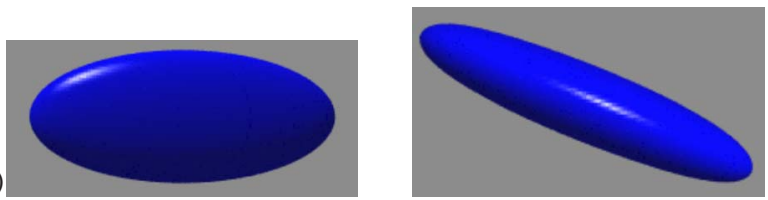

(b)
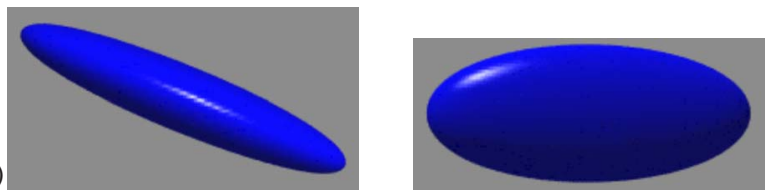

(c)
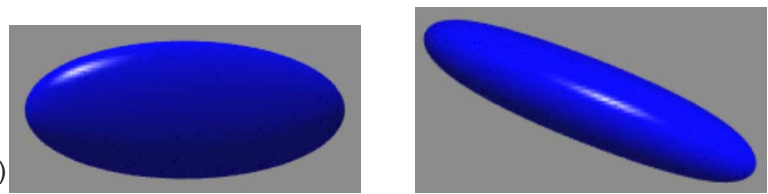

FIG. 5. (Color online) Numerical results for an initial interval of time where the slope in Fig. 4 is steep. (a) $t / \tau_{\mathrm{Ca}}=0.27$, (b) $t / \tau_{\mathrm{Ca}}$ $=0.5$, and (c) $t / \tau_{C a}=0.8 . S=2, K=1, \tau_{C a}=0.56, \tau_{1} / \tau_{C a}=9.62$, and $\tau_{2} / \tau_{C a}=2.19$. Top view and side view. 
(a)

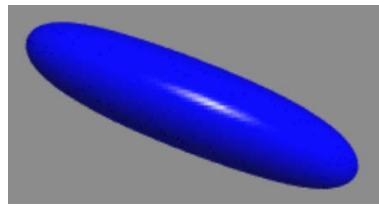

(b)
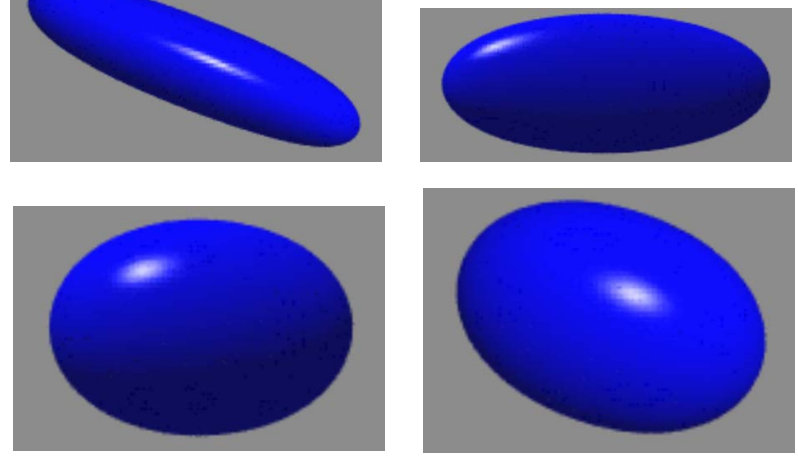

FIG. 6. (Color online) Numerical results at (a) $t / \tau_{C a}=1.07$ and (b) $t / \tau_{C a}=6.2 . S=2, K=1, \tau_{C a}=0.56, \tau_{1} / \tau_{C a}=9.62$, and $\tau_{2} / \tau_{C a}$ $=2.19$. Top view and side view.

of the drop shape. The transition between the two regimes of drop evolution is not sharp enough to pinpoint a precise transition point, but the value 0.34 of the Hencky strain given in [1] lies within the transition interval.

Figure 4 shows the evolution of the log of the difference between the maximum and minimum curvature values at each time $t / \tau_{C a}$. We see an initial steep decrease, followed by two roughly linear regimes. The slope in the late stage of the evolution ought to be $1 / \tau_{2}$ as indicated by the dashed line; we actually see a somewhat flatter slope, probably because the numerical results become inaccurate when the difference between maximum and minimum curvature is small. We can try to predict the slope in the earlier regime based on a priori assumptions on the drop shape. If we assume the drop has length $L$ and $L$ is large, then, for an axisymmetric ellipsoid, the maximum curvature is of order $L^{2}$, and for a cylinder with spherical caps it is of order $\sqrt{L}$. Hence, if $L$ behaves like $\exp \left(-t / \tau_{1}\right)$, then the curvature difference should behave like

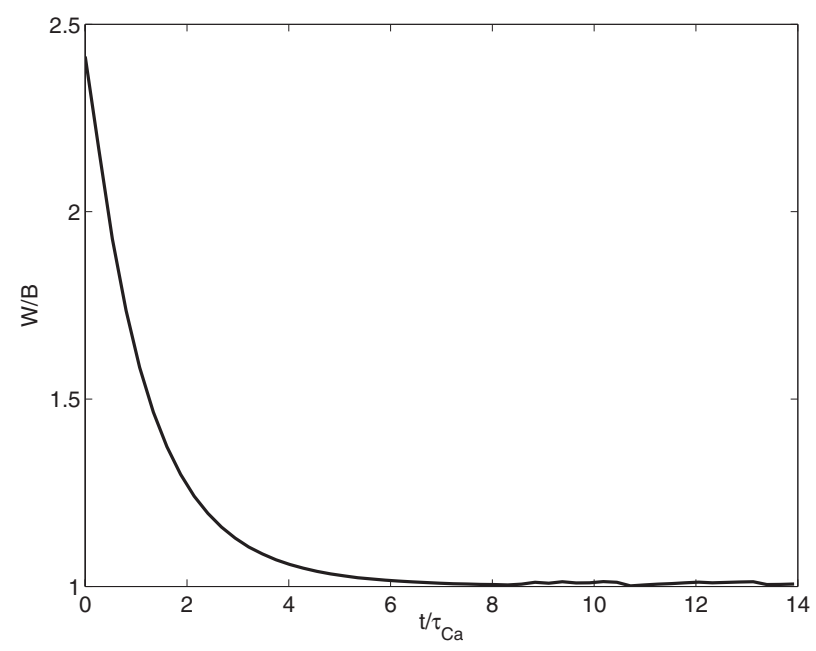

FIG. 7. Numerical results for the ratio of top-view width $W$ to side-view width $B . S=2, K=1, \tau_{C a}=0.56, \tau_{1} / \tau_{C a}=9.62$, and $\tau_{2} / \tau_{C a}=2.19$.

$\exp \left[-t /\left(2 \tau_{1}\right)\right]$ for a cylinder with spherical caps and like $\exp \left(-2 t / \tau_{1}\right)$ for an axisymmetric ellipsoid. A line of slope $-2 / \tau_{1}$ has been included in the figure; we see that even that slope is too flat to fit our results. We conclude that the assumption of fixed drop shape is too simplistic to fit our results, and the curvature evolution probably reflects changes in drop shape rather than simply retraction with a given shape.

Drop shapes are helpful in clarifying the initial stage of evolution. In Figs. 5 and 6, we show top-view and side-view pictures of the drop in the initial time interval, where the curvature difference decreases rapidly. We see that in this initial interval, the drop evolves from a "flattened pancake" shape to an axisymmetric shape. The top-view width $W$ decreases during the evolution toward axisymmetry and then

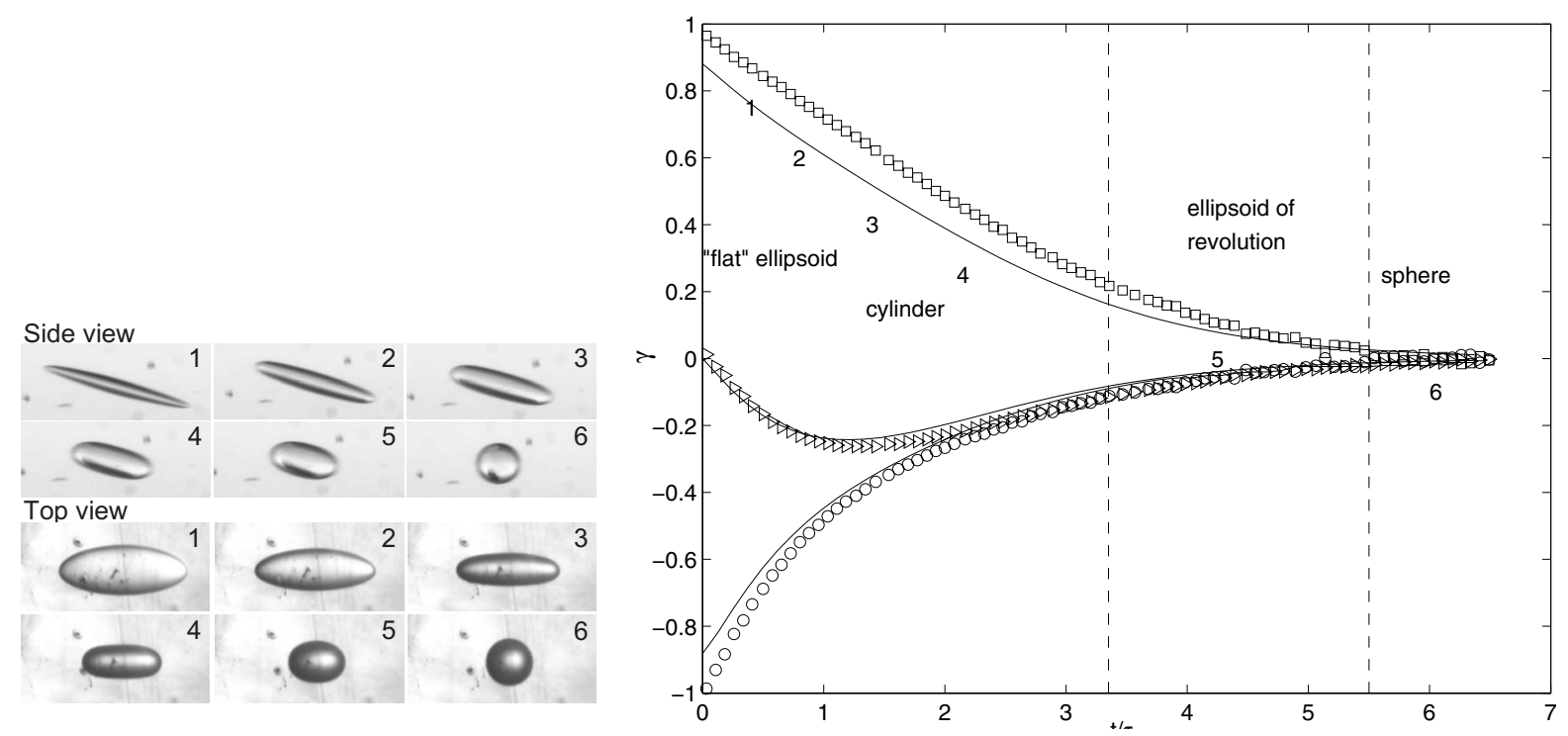

FIG. 8. Comparison of numerical results with experimental data for $\gamma=\ln \left(L / 2 r_{0}\right)(\square), \ln \left(B / 2 r_{0}\right)(\bigcirc)$, and $\ln \left(W / 2 r_{0}\right)(\triangleright)$ for the side-view length $L$ and breadth $B$, and top-view width $W$. Numerical results are lines, computed at $S=2, K=1, \tau_{C a}=0.56, \tau_{1} / \tau_{C a}=9.62$, and $\tau_{2} / \tau_{C a}$ $=2.19$. Experimental data and photographs are at $K=0.01$, macroscopic strain $S \approx 2$, for a drop radius $r_{0} \approx 200-300$ micron, $\tau_{2} / \tau_{C a}=1.21$ $[1,5]$. 
increases again once an axisymmetric shape is reached. We can get an idea of the transition time from Fig. 7, which shows the ratio of top-view width $W$ to side-view width $B$. We find this time to be not entirely negligible, on the order of twice the capillary time.

There is excellent agreement with measured results of side-view length $L(\square)$ and width $B(\bigcirc)$ and top-view width $W(\triangleright)$ given in Fig. 8. The experimental conditions are detailed in $[1,5]$. Numerical results are lines superposed on the data. The initial condition for the drop is not quite the same in the simulation and in the experiments. For the simulation, we subjected the drop to a shear of magnitude 2 from its spherical configuration. In the experiments, this shear is applied to plates at some distance from the drop, and the drop does not quite deform affinely with the external flow, but actually reaches a slightly larger initial length. The numbers in the figure correspond to the experimental photographs and phases in the drop shapes: "flat" ellipsoid, cylinder, ellipsoid of evolution, and sphere. The transition between the flat el- lipsoid and cylinder occurs when the top-view width curves around the minimum value.

\section{CONCLUSIONS}

We find a two-step relaxation process, with relaxation times in agreement with the experimental findings. We also find that the hypothesis of exponential decay of curvatuves is appxoximately valid. However, the actual decay rate of curvature is not consistent with the heuristics advanced in [1].

\section{ACKNOWLEDGMENTS}

This research was supported by the National Science Foundation under Grants No. DMS-0707727, No. DMS0405810, and No. DMS-0456086, NCSA Grant No. TGCTS060013N, and by Le Conseil Régional des Pays de la Loire.
[1] S. Assighaou and L. Benyahia, Phys. Rev. E 77, 036305 (2008).

[2] Y. Renardy and M. Renardy, J. Comput. Phys. 183, 400 (2002).

[3] R. Scardovelli and S. Zaleski, Annu. Rev. Fluid Mech. 31, 567
(1999).

[4] J. U. Brackbill, D. B. Kothe, and C. Zemach, J. Comput. Phys. 100, 335 (1992).

[5] S. Assighaou, Ph.D. thesis, University of Le Mans, Le Mans, France, 2006. 